International Mathematical Forum, 2, 2007, no. 24, 1163 - 1169

\title{
Band Congruences in Ordered Semigroups
}

\author{
Niovi Kehayopulu and Michael Tsingelis \\ Department of Mathematics \\ University of Athens \\ 157 84, Panepistimiopolis, Athens, Greece \\ nkehayop@math.uoa.gr
}

\begin{abstract}
The semilattice congruences play an important role in studying the decomposition of semigroups -without order. When we pass from semigroups without order to ordered semigroups, the same role is played by the complete semilattice congruences. So, the complete semilattice congruences play an essential role in studying the structure, especially the decomposition of ordered semigroups. The characterization of complete semilattices of semigroups of a given type has been already considered by the same authors. Band congruences have been also proved to be useful in studying the decomposition of some types of ordered semigroups, especially the decomposition of $t$-archimedean ordered semigroups. In this paper we prove that an ordered semigroup $S$ is a band of semigroups of a given type $\mathcal{T}$ if and only if it is decomposable into pairwise disjoint subsemigroups $S_{\alpha}$ of $S$ of type $\mathcal{T}$ indexed by a band $B$, such that $S_{\alpha} S_{\beta} \subseteq S_{\alpha \beta}$ for all $\alpha, \beta \in B$ and $S_{\alpha} \cap\left(S_{\beta}\right] \neq \emptyset$ implies $\alpha=\alpha \beta=\beta \alpha$.
\end{abstract}

\section{Mathematics Subject Classification: 06F05}

Keywords: Ordered semigroup, congruence, band, semilattice congruence, complete semilattice congruence, band congruence, band of semigroups of type $\mathcal{T}$.

\section{Introduction-Preliminary Notes}

The semilattice congruences play an important role in studying the structure, especially the decomposition of semigroups -without order (cf., for example, [1],[5]). In case of ordered semigroups, the same role is played by the complete semilattice congruences. So the complete semilattice congruences play an important role in studying the structure, especially the decomposition of ordered semigroups. The characterization of complete semilattices of semigroups of a 
given type has been already considered by the same authors in [3]. It has been proved in [3] that an ordered semigroup $S$ is a complete semilattice of semigroups of a given type, say $\mathcal{T}$, if and only if it is decomposable into pairwise disjoint subsemigroups $S_{\alpha}$ of $S$ of type $\mathcal{T}$, indexed by a semilattice $Y$ in such a way that: (1) $S_{\alpha} S_{\beta} \subseteq S_{\alpha \beta}$ for all $\alpha, \beta \in Y$ and (2) If $\alpha, \beta \in Y$ such that $S_{\alpha} \cap\left(S_{\beta}\right] \neq \emptyset$, then $\alpha=\alpha \beta(=\beta \alpha)$. Band congruences have been proved to be useful in studying the structure of some ordered semigroups referring to the decomposition as, for example, the decomposition of some types of ordered semigroups into $t$-archimedean components [4]. Since a semilattice is a commutative semigroup, the problem in case of complete semilattice congruences is more natural, since in that case the semilattice $(Y,$.$) endowed with the order$ $\preceq:=\{(\alpha, \beta) \mid \alpha=\alpha \beta(=\beta \alpha)\}$ is an ordered semigroup. However, this is not the case for band congruences, for which the corresponding relation " $\preceq$ " is just an order and the band endowed with the order " $\preceq "$ is not an ordered semigroup (unless in the special case for which the band is a semilattice). In this paper we prove that an ordered semigroup $S$ is a band of semigroups of a given type $\mathcal{T}$ if and only if it is decomposable into pairwise disjoint subsemigroups $S_{\alpha}$ of $S$ of type $\mathcal{T}$, indexed by a band $B$, such that (1) $S_{\alpha} S_{\beta} \subseteq S_{\alpha \beta}$ for all $\alpha, \beta \in B$ and (2) If $\alpha, \beta \in B$ such that $S_{\alpha} \cap\left(S_{\beta}\right] \neq \emptyset$, then $\alpha=\alpha \beta=\beta \alpha$.

If $S$ is an ordered semigroup, an equivalence relation $\sigma$ on $S$ is called a right (resp. left) congruence on $S$ if $(a, b) \in \sigma$ implies $(a c, b c) \in \sigma(\operatorname{resp} .(c a, c b) \in \sigma)$ for all $c \in S$. It is called a congruence on $S$ if it is both a right and a left congruence on $S$. A congruence $\sigma$ on $S$ having the properties $\left(a, a^{2}\right) \in \sigma$ and $(a b, b a) \in \sigma$ for all $a, b \in S$, is called a semilattice congruence on $S$. A semilattice congruence $\sigma$ on $S$ is called complete if $a \leq b$ implies $(a, a b) \in \sigma$. In this paper, we first introduce the concept of a band congruence: A congruence $\sigma$ on an ordered semigroup $(S, ., \leq)$ is called a band congruence if $a \leq b$ implies $(a, a b) \in \sigma$ and $(a, b a) \in \sigma$. One can easily see that a complete semilattice congruence is a band congruence. This is because a complete semilattice congruence $\sigma$ has the property $(a b, b a) \in \sigma$ for all $a, b \in S$. This together with the transitivity relation of $\sigma$ shows that $\sigma$ is a band congruence. An ordered semigroup $S$ is called a band of semigroups of a given type, say $\mathcal{T}$, if there exists a band congruence $\sigma$ on $S$ such that the $\sigma$-class $(x)_{\sigma}$ of $S$ is a subsemigroup of $S$ of type $\mathcal{T}$ for each $x \in S$. A semigroup $(S,$.$) is called a band if every element$ of $S$ is idempotent, i.e. $a^{2}=a$ for every $a \in S$. If $(S,$.$) is a semigroup and the$ set $E_{S}$ of idempotent elements of $S$ is nonempty, then the $E_{S}$ with the relation "§" on $E_{S}$ defined by

$$
\preceq:=\{(e, f) \in S \times S \mid e=e f=f e\}
$$

is an ordered set. So if a semigroup $(S,$.$) is a band, then the set S$ endowed with the relation

$$
\preceq:=\{(x, y) \in S \times S \mid x=x y=y x\}
$$


(that is $x \preceq y$ if and only if $x=x y=y x$ ) is an ordered set (cf. also [1, p.24]). We recall that if $(S,$.$) is a band, then (S, . \preceq)$ is not an ordered semigroup, in general (unless in the special case when the multiplication on $S$ is commutative). In any case, the present paper deals with pure bands. This is because if a band is commutative, then we deal with a semilattice, and the problem in that case has been already proved in [3]. In the following when we refer to an order on a band $B$, we always mean the order " $\preceq$ " on $B$ defined by

$$
a \preceq b \text { if and only if } a=a b=b a .
$$

If $(S, ., \leq)$ is an ordered semigroup and $H \subseteq S$, we denote by $(H]$ the subset of $S$ defined by

$$
(H]:=\{t \in S \mid t \leq h \text { for some } h \in H\} .
$$

For ordered semigroups the congruences (and the semilattice congruences) are defined exactly as in semigroups without order. For a semigroup (or an ordered semigroup) $S$, we denote by $(x)_{\sigma}$ the $\sigma$-class of $S$ containing $x(x \in S)$. It is well known that if $S$ is a semigroup and $\sigma$ a congruence on $S$, then the set $S / \sigma:=\left\{(x)_{\sigma} \mid x \in S\right\}$ with the multiplication "o" on $S / \sigma$ defined by $(x)_{\sigma} \circ(y)_{\sigma}:=(x y)_{\sigma}$ is a semigroup (cf. [5],[2]). In the sequel, the multiplication on $S / \sigma$ is denoted by " $\circ "$.

\section{Main Results}

Definition 2.1. Let $(S, ., \leq)$ be an ordered semigroup. A band congruence on $S$ is a congruence $\sigma$ on $S$ such that

$$
a \leq b \Longrightarrow(a, a b) \in \sigma \text { and }(a, b a) \in \sigma
$$

Remark 2.2. Let $(S, ., \leq)$ be an ordered semigroup and $\sigma$ a band congruence on $S$. Then we have the following:

(1) $\left(a, a^{2}\right) \in \sigma$ and $\left(a^{2}, a\right) \in \sigma$ for all $a \in S$. Indeed: If $a \in S$, then $a \leq a$, so $\left(a, a^{2}\right) \in \sigma$. Since $\sigma$ is symmetric, we also have $\left(a^{2}, a\right) \in \sigma$.

(2) $(a)_{\sigma} \circ(a)_{\sigma}=(a)_{\sigma}$ for all $a \in S$. Indeed: If $a \in S$, then by (1), we have

$(a)_{\sigma} \circ(a)_{\sigma}:=\left(a^{2}\right)_{\sigma}=(a)_{\sigma}$.

(3) $(S / \sigma, \circ)$ is a band. Indeed: As we have already seen $(S / \sigma, \circ)$ is a semigroup, and by (2), it is a band.

(4) $(a)_{\sigma}$ is a subsemigroup of $S$ for all $a \in S$. Indeed: Let $a \in S$. Clearly, $\emptyset \neq(a)_{\sigma} \subseteq S$. Let $x, y \in(a)_{\sigma}$. Then $(x, a) \in \sigma,(y, a) \in \sigma$. Since $\sigma$ is a congruence on $S$, we have $\left(x y, a^{2}\right) \in \sigma$. By $(1),\left(a^{2}, a\right) \in \sigma$. Thus we have $(x y, a) \in \sigma$, and $x y \in(a)_{\sigma}$.

(5) $(a)_{\sigma}(b)_{\sigma} \subseteq(a b)_{\sigma}$ for all $a, b \in S$. Indeed: Let $a, b \in S, x \in(a)_{\sigma}$ and $y \in(b)_{\sigma}$. Since $(x, a) \in \sigma$ and $(y, b) \in \sigma$, we get $(x y, a b) \in \sigma$, so $x y \in(a b)_{\sigma}$. 
(6) $(a)_{\sigma}(b)_{\sigma} \subseteq(a)_{\sigma} \circ(b)_{\sigma}$ for all $a, b \in S$. Indeed: If $a, b \in S$ then, by (5), we have $(a)_{\sigma}(b)_{\sigma} \subseteq(a b)_{\sigma}:=(a)_{\sigma} \circ(b)_{\sigma}$.

(7) If $a \leq b$, then $(a b, b a) \in \sigma$. Indeed: Let $a \leq b$. Since $\sigma$ is a band congruence on $S$, we have $(a, a b) \in \sigma$ and $(a, b a) \in \sigma$. Since $\sigma$ is a congruence, it is symmetric, so we have $(a b, a) \in \sigma$. Then, since $\sigma$ is transitive, we have $(a b, b a) \in \sigma$.

Remark 2.3. If $\sigma$ is a band congruence on an ordered semigroup $(S, ., \leq)$ then, as we have already seen in Remark $2.2,(S / \sigma, \circ)$ is a band. So the set $S / \sigma$ with the relation " $\preceq "$ on $S / \sigma$ defined by:

$$
\begin{aligned}
(a)_{\sigma} \preceq(b)_{\sigma} & \Longleftrightarrow(a)_{\sigma}=(a)_{\sigma} \circ(b)_{\sigma}=(b)_{\sigma} \circ(a)_{\sigma} \\
& \Longleftrightarrow(a)_{\sigma}=(a b)_{\sigma}=(b a)_{\sigma} \\
& \Longleftrightarrow(a, a b) \in \sigma \text { and }(a, b a) \in \sigma
\end{aligned}
$$

is an ordered set. Moreover, since $\sigma$ is a band congruence, we have

$$
\begin{aligned}
a \leq b & \Longrightarrow(a, a b) \in \sigma \text { and }(a, b a) \in \sigma \\
& \Longrightarrow(a)_{\sigma} \preceq(b)_{\sigma} .
\end{aligned}
$$

Definition 2.4. Let $(A,),.(B, *)$ be two groupoids and " $\leq_{A} ", " \leq_{B}$ " order relations on $A$ and $B$, respectively. A mapping $f:\left(A, . \leq_{A}\right) \rightarrow\left(B, *, \leq_{B}\right)$ is called a homomorphism if

(1) $f(x y)=f(x) * f(y)$ for all $x, y \in A$

(2) If $x \leq_{A} y$, then $f(x) \leq_{B} f(y)$.

Definition 2.5. Let $(S, ., \leq)$ be an ordered semigroup. $S$ is called a band of semigroups of type $\mathcal{T}$, if there exists a band congruence $\sigma$ on $S$ such that the $\sigma$-class $(a)_{\sigma}$ is a subsemigroup of $S$ of type $\mathcal{T}$ for every $a \in S$.

Theorem 2.6. Let $(S, ., \leq)$ be an ordered semigroup. The following are equivalent:

(1) $S$ is a band of semigroups of type $\mathcal{T}$.

(2) There exists a band $(B, *)$ and a mapping $f:(S, ., \leq) \rightarrow(B, *, \preceq)$ which is homomorphism and onto, such that $f^{-1}(\{\beta\})$ is a subsemigroup of $S$ of type $\mathcal{T}$ for every $\beta \in B$.

(3) There exists a band $(B, *)$ and a homomorphism $f:(S, ., \leq) \rightarrow(B, *, \preceq)$ such that $f^{-1}(\{\beta\})$ is a subsemigroup of $S$ of type $\mathcal{T}$ for every $\beta \in B$.

(4) There exists a band $(B, *)$ and a family $\left\{S_{\beta}\right\}_{\beta \in B}$ of subsemigroups of $S$ of type $\mathcal{T}$, such that

(I) $S_{\alpha} \cap S_{\beta}=\emptyset$ for all $\alpha, \beta \in B, \alpha \neq \beta$

(II) $S=\bigcup_{\alpha \in B} S_{\alpha}$ 
(III) $S_{\alpha} S_{\beta} \subseteq S_{\alpha * \beta}$ for all $\alpha, \beta \in B$

(IV) If $\alpha, \beta \in B$ such that $S_{\alpha} \cap\left(S_{\beta}\right] \neq \emptyset$, then $\alpha \preceq \beta$ (i.e. $\alpha=\alpha * \beta=\beta * \alpha$ ).

Proof. $(1) \Longrightarrow(2)$. Let $\sigma$ be a band congruence on $S$ such that $(x)_{\sigma}$ is a subsemigroup of $S$ of type $\mathcal{T}$ for every $x \in S$. As we have already seen in Remark $2.2,(S / \sigma, \circ)$ is a band. We consider the mapping:

$$
f:(S, ., \leq) \rightarrow(S / \sigma, \circ, \preceq) \mid x \rightarrow(x)_{\sigma} .
$$

The mapping $f$ is a homomorphism. Indeed: If $x, y \in S$, then $f(x y):=$ $(x y)_{\sigma}:=(x)_{\sigma} \circ(y)_{\sigma}=f(x) \circ f(y)$. If $x \leq y$, then $(x)_{\sigma} \preceq(y)_{\sigma}$ (cf. Remark 2.3). The mapping $f$ is clearly an onto mapping.

Let now $\beta \in S / \sigma$ and let $x \in S$ such that $(x)_{\sigma}=\beta$. We have

$$
y \in f^{-1}(\{\beta\}) \Longleftrightarrow f(y)=\beta \Longleftrightarrow(y)_{\sigma}=(x)_{\sigma} \Longleftrightarrow y \in(x)_{\sigma}
$$

that is, $f^{-1}(\{\beta\})=(x)_{\sigma}$. So $f^{-1}(\{\beta\})$ is a subsemigroup of $S$ of type $\mathcal{T}$ for every $\beta \in S / \sigma$, and (2) is satisfied.

$(2) \Longrightarrow(3)$. It is obvious.

$(3) \Longrightarrow(4)$. Let $(B, *)$ be a band and $f:(S, ., \leq) \rightarrow(B, *, \preceq)$ be a homomorphism such that $f^{-1}(\{\beta\})$ is a subsemigroup of $S$ of type $\mathcal{T}$ for every $\beta \in B$. For each $\beta \in B$, we put $S_{\beta}:=f^{-1}(\{\beta\})$. Then we have the following:

(I) Let $\alpha, \beta \in B, \alpha \neq \beta$. Then $f^{-1}(\{\alpha\}) \cap f^{-1}(\{\beta\})=\emptyset$. Indeed:

If $t \in f^{-1}(\{\alpha\}) \cap f^{-1}(\{\beta\})$, then $f(t)=\alpha, f(t)=\beta$, so $\alpha=\beta$ which is impossible.

(II) $S=\bigcup_{\alpha \in B} S_{\alpha}$. Indeed: If $\alpha \in B$, then $\{\alpha\} \subseteq B$, so $f^{-1}(\{\alpha\}) \subseteq S$ for every $\alpha \in B$, and $\bigcup_{\alpha \in B} S_{\alpha} \subseteq S$. Let now $x \in S$. Then, for the element $\alpha:=f(x) \in B$, we have $f(x)=\alpha$. So $x \in f^{-1}(\{\alpha\}) \subseteq \bigcup_{\alpha \in B} S_{\alpha}$.

(III) Let $\alpha, \beta \in B$. Then $f^{-1}(\{\alpha\}) f^{-1}(\{\beta\}) \subseteq f^{-1}(\{\alpha * \beta\})$. Indeed:

Let $x y \in f^{-1}(\{\alpha\}) f^{-1}(\{\beta\})$, where $x \in f^{-1}(\{\alpha\}), y \in f^{-1}(\{\beta\})$. Since $f$ is a homomorphism, we have $f(x y)=f(x) * f(y)=\alpha * \beta$, so $x y \in f^{-1}(\{\alpha * \beta\})$. (IV) Let $\alpha, \beta \in B$ such that $S_{\alpha} \cap\left(S_{\beta}\right] \neq \emptyset$. Then $\alpha \preceq \beta$. Indeed: Let $x \in S_{\alpha} \cap\left(S_{\beta}\right]$. Since $x \in S_{\alpha}:=f^{-1}(\{\alpha\})$, we have $f(x)=\alpha$. Since $x \in\left(S_{\beta}\right]$, there exists $y \in S_{\beta}$ such that $x \leq y$. Since $y \in S_{\beta}:=f^{-1}(\{\beta\})$, we have $f(y)=\beta$. On the other hand, since $x \leq y$ and $f$ is a homomorphism, we have $f(x) \preceq f(y)$. Therefore we have $\alpha \preceq \beta$.

$(4) \Longrightarrow(1)$. We define a relation " $\sigma$ " on $S$ as follows:

$$
\sigma:=\left\{(x, y) \in S \times S \mid \exists \alpha \in B: x, y \in S_{\alpha}\right\} .
$$

Then we have the following:

(A) $\sigma$ is a band congruence on $S$. In fact: 
If $x \in S\left(=\bigcup_{\alpha \in B} S_{\alpha}\right)$, then there exists $\alpha \in B$ such that $x \in S_{\alpha}$, thus $(x, x) \in \sigma$. The relation $\sigma$ is clearly symmetric.

Let $(x, y) \in \sigma,(y, z) \in \sigma$. Then there exists $\alpha \in B$ such that $x, y \in S_{\alpha}$ and $\beta \in B$ such that $y, z \in S_{\beta}$. If $\alpha \neq \beta$, then $y \in S_{\alpha} \cap S_{\beta}=\emptyset$ which is impossible. Thus we have $\alpha=\beta$, then $S_{\alpha}=S_{\beta}$, and $x, y \in S_{\alpha}$. Therefore we have $(x, z) \in \sigma$.

Let $(x, y) \in \sigma, z \in S$. Then $(x z, y z) \in \sigma$. Indeed: Since $(x, y) \in \sigma$, there exists $\alpha \in B$ such that $x, y \in S_{\alpha}$. Since $z \in S$, we have $z \in S_{\beta}$ for some $\beta \in B$. By hypothesis, we have $x z, y z \in S_{\alpha} S_{\beta} \subseteq S_{\alpha * \beta}$, where $\alpha * \beta \in B$, so $(x z, y z) \in \sigma$.

In a similar way we prove that $\sigma$ is a left congruence on $S$.

Let $x \leq y$. Then $(x, x y) \in \sigma$ and $(x, y x) \in \sigma$. Indeed: Since $x \in S$, there exists $\alpha \in B$ such that $x \in S_{\alpha}$. Since $y \in S$, there exists $\beta \in B$ such that $y \in S_{\beta}$. Since $S \ni x \leq y \in S_{\beta}$, we have $x \in\left(S_{\beta}\right]$. Hence we have $x \in S_{\alpha} \cap\left(S_{\beta}\right]$. Then, by (IV), we get $\alpha \preceq \beta$ i.e. $\alpha=\alpha * \beta=\beta * \alpha$. By (III), we have $x y \in S_{\alpha} S_{\beta} \subseteq S_{\alpha * \beta}=S_{\alpha}$ and $y x \in S_{\beta} S_{\alpha} \subseteq S_{\beta * \alpha}=S_{\alpha}$. Since $x, x y \in S_{\alpha}$, $\alpha \in B$, we have $(x, x y) \in \sigma$. Since $x, y x \in S_{\alpha}, \alpha \in B$, we have $(x, y x) \in \sigma$. Therefore the relation $\sigma$ is a band congruence on $S$.

(B) Let $x \in S$. Then $(x)_{\sigma}$ is a subsemigroup of $S$ of type $\mathcal{T}$. In fact:

Let $x \in S_{\alpha}$ for some $\alpha \in B$. We have $(x)_{\sigma}=S_{\alpha}$. Indeed: Let $y \in(x)_{\sigma}$. Since $(y, x) \in \sigma$, there exists $\beta \in B$ such that $y, x \in S_{\beta}$. If $\alpha \neq \beta$, then $x \in S_{\alpha} \cap S_{\beta}=\emptyset$ which is impossible. Thus we have $\alpha=\beta$, and $y \in S_{\alpha}$. Let now $y \in S_{\alpha}$. Since $x, y \in S_{\alpha}$, where $\alpha \in B$, we have $(x, y) \in \sigma$, then $y \in(y)_{\sigma}=(x)_{\sigma}$.

Remark 2.7. In Theorem 2.6 condition (2) is stated only for emphasis. Clearly if $f^{-1}(\{\beta\})$ is a subsemigroup of $S$ for every $\beta \in B$, then $f^{-1}(\{\beta\}) \neq \emptyset$ $\forall \beta \in B$, and so the mapping $f$ is onto.

\section{ACKNOWLEDGMENTS}

We thank Professor Josef S. Ponizovskii for reading the paper carefully before submitting it and the useful discussions concerning this paper we had. We also thank the Managing Editor of the journal Professor Emil Minchev for editing and communicating the paper.

This research has been supported by the Special Research Account of the University of Athens, grant no. 70/4/5630.

\section{References}

[1] A. H. Clifford and G. B. Preston, The Algebraic Theory of Semigroups, Vol. I, Amer. Math. Soc., Math. Surveys 7, Providence, Rhode Island, 1964. 
[2] N. Kehayopulu, On right regular and right duo ordered semigroups, Mathematica Japonica 36, no. 2 (1991), 201-206.

[3] N. Kehayopulu, M. Tsingelis, A remark on semilattice congruences in ordered semigroups (Russian), Izv. Yyssh. Uchebn. Zaved. Mat. 2000, no. 2, 50-52; translation in Russian Math. (Iz. VUZ) 44 (2000), no. 2, 48-50.

[4] N. Kehayopulu, M. Tsingelis, Band congruences of $t$-archimedean ordered semigroups, Algebra Colloquium, to appear.

[5] M. Petrich, Introduction to Semigroups, Merrill Research and Lecture Series. Charles E. Merrill Publishing Company, A Bell and Howell Company, Columbus, Ohio, 1973.

Received: June 21, 2006 